\title{
A Prospective Analysis of Intrathecal Granuloma in Chronic Pain Patients: A Review of the Literature and Report of A SURVEILlance Study
}

\author{
Timothy R. Deer, MD
}

\begin{abstract}
Background: Over the past decade granulomas have been noted to occur at or near the tip of intrathecal catheters used for spinal infusions. The majority of cases involved morphine infusions, although other drugs have been implicated. Granulomas may be asymptomatic or cause significant neurological deficits.

Methods: Consecutive patients with intrathecal infusions were examined for the presence of intrathecal granulomas. MRI or CT was used to make the diagnosis.

Results: A total of 208 patients under-
\end{abstract}

went imaging over a period of 34 weeks. Six patients (3\%) were found to have a significant lesion. Five of the six patients were asymptomatic and one patient complained of radicular pain in the distribution of the catheter tip. The average catheter infusion time for patients with granulomas was 27 months, not significantly different from patients without granulomas. No specific characteristics, such as drugs or concentrations were identified. All six patients had percutaneous catheter revisions without complication.

Conclusion: Intrathecal granulomas were identified in $3 \%$ of patients imaged in this series. Eighty percent of the patients were asymptomatic. MRI imaging remains the diagnostic method of choice for most patients, and can be done safely when scans are taken at the level of the catheter tip. Given the low incidence of granulomas with intrathecal catheters, routine imaging to identify granulomas is not warranted.

Keywords: Intrathecal granulomas, intrathecal inflammatory masses, intrathecal catheters, intrathecal infusion pumps
Intrathecal infusions place drugs in direct contact with the central nervous system. This route of therapy has been shown to be efficacious in many studies involving both cancer and non-cancer pain syndromes (1). The most commonly described side effects and complications have involved nausea, urinary retention, itching, headache, peripheral edema and drowsiness. Classic studies of outcomes do not report inflammatory mass or granuloma formation as a problem (2). In 1991, North and colleagues (3) described the formation of a granuloma at the catheter tip causing neurological difficulties. When this article was published it was felt by many to be an interesting and rare event that was not clinically significant.

However, recently published literature and data reports to the Food and Drug Administration suggest the prevalence of this problem may be more worrisome than originally thought. Burchiel and Coffey (4) reported an increase in the

From West Virginia University School of Medicine, Charleston, West Virginia. Address Correspondence: Timothy R. Deer, MD, The Center for Pain Relief, 400 Court Street, Suite 302, Charleston, West Virginia 25301.

E-mail: jgoodwin@centerforpainrelief.com number of reported cases over the past three years, and theorized that the problem is worse than previously thought because of lack of physician reporting.

Sequelae of granuloma range from paralysis to incidental findings that are asymptomatic. The unknown significance and true prevalence of this problem has led many to theorize that the inflammatory mass will lead to reduction in the use of this therapy. In order to better understand the frequency of occurrence of this problem an analysis of 208 consecutive patients with MRI or CT myelogram who presented for pump refill was conducted.

\section{OVERVIEW OF GRANULOMAS}

\section{Clinical Prevalence and Presentation}

Some authors (5) have reported granulomas to occur in close to $50 \%$ of their patients, however that report involved a small sample of patients in a single practice. Other reports have varied from $0.1 \%$ to $5 \%$ of patients $(4,5,13,15$, 17). This wide range of reports suggests the true prevalence is unknown. In some cases, clusters of granulomas have been reported suggesting a definable cause. The duration of infusion therapy exposure until granuloma diagnosis is made is reported to vary from 0.5 to 72 months, with an average time of 24 months until the problem is identified. The diagnosis of this problem often is made after neurological symptoms or signs develop (6).

The most common reported presentations have been loss of effective pain relief, frequent need for dose escalation, dermatomal symptoms in the distribution of the catheter tip, higher than predicted residual volume remaining in the reservoir at pump refills, change in proprioception, change in sensation, and in late stages, motor loss and bladder and bowel symptoms. Physical exam may initially be normal, but may involve changes in sensation, reflexes, proprioception or motor function. Any change of motor function in the presence of a MRI proven inflammatory mass should warrant an immediate spine surgery consultation (6).

\section{Etiology of Granulomas}

Intrathecal granulomas appear to be related to an inflammatory reaction to opioids that ceases once the offending drug is discontinued. Theories relating to granuloma formation have included allergies to silicone catheters and reactions to impurities in compounded drugs. Both of these theories appear to be incor- 
rect. There have been no reported cases of granuloma formation in baclofen infusion patients with identical silicone catheters. There is no documented difference in the occurrence of granulomas in patients receiving compounded drugs when compared to manufactured medication $(16,17)$.

The literature suggests that the etiology of granulomas may be related to a mitogen-activated protein kinase cascade that causes increased lymphocyte activity and changes in mast cell activity (69). Granulomas have been reported most commonly with morphine. Other drugs have also been implicated in causing this complication, including hydromorphone and fentanyl $(4,6,13)$. Clonidine has been shown in dog models to provide some protection against granuloma, but this has not been proven in clinical studies (6) The concentration of opioid infused appears to play some role in this process. Despite this finding there have been reported cases of inflammatory mass at low concentrations of morphine and hydromorphone, which suggests the need for vigilance in all patients $(4,6,10)$.

The proposed increase in the occurrence of granulomas in single exit catheters has been theorized to occur due to increased drug concentrations with this type of flow. The absence of reported occurrences of granulomas in wire reinforced catheters used for constant flow pumps has also led to the suggestion that this type of system may be protective, although no studies support this statement at present.

The most common location for diagnosis of granuloma is the thoracic spine. The ventral thoracic subarachnoid space has the longest region of low CSF flow within the entire intrathecal compartment (12). This stagnation of flow has been postulated to cause buildup of the offending drug, causing a peak in both concentration and total dose. This conclusion may be faulty because of the high percentage of indwelling catheters that are placed in this location. Although another theory regarding granuloma formation is the possibility of an infectious process, tissue samples in both animals and patients undergoing tissue resection have shown no evidence of infection $(4,6)$.

\section{Diagnosing Granulomas}

MRI is the gold standard for diagnosing intrathecal granulomas. MRI can be used safely when the magnet strength is less than 2.0 Tesla; testing has not been reported using stronger magnetic fields. The magnetic field can produce a small tug on the pump, but the effect is less than that due to gravity. There may also be a small amount of tissue heating adjacent to the pump, on the order $1^{\circ} \mathrm{C}$. The magnetic field will stop the rotation of the pump rotor, so the patient will not receive drug during the scan. Although it is not necessary to stop the pump, it may be turned off during the scan. In either case, the computer pump programmer should be used after the scan to verify that no changes in programming occurred, although such an event is considered unlikely for the SynchroMed pump (Medtronic Neurological, Minneapolis, Minnesota; see website information at www.medtronic.com).

When MRI is not possible CT myelogram is the alternate test of choice. In asymptomatic patients an imaging study can be performed as part of routine analysis of the pump system. Patients should undergo an initial neurological examination prior to pump implant, and then intermittent exams at times of pump refill. Changes in symptoms or physical examination should lead to suspicion of granuloma, although the etiology of these problems may be multiple and could include disease progression, lumbar disc herniation, new disease processes, catheter malfunction, and pump malfunction.

The study of choice in patients in whom the possibility of a granuloma is considered is a T1 weighted MRI. The addition of gadolinium may give additional information, but does not appear to be critical in diagnosis. In a few reported cases, side port studies have also been helpful in visualizing and diagnosing granuloma. Pain on injecting or failure to successfully aspirate the side port are possible warning signs of tip granuloma $(5,6$, $13,14,20)$.

\section{Treatment of Granulomas}

Once a granuloma or inflammatory mass is diagnosed, the physician must determine the appropriate clinical response. The majority of reported cases have been treated by conservative measures. A neurosurgical or orthopedic consultation may be required if signs of impending neurological loss are present or if the diagnostic images suggest significant spinal cord compression.

\section{Conservative Treatment}

If the finding is occult and the patient has not experienced symptoms, the response can be conservative. This would include discontinuation of the infused drug and changing to a different opioid or non-opioids. If this approach is chosen, close clinical follow-up and serial diagnostic images should be performed. In rare instances regression of the granuloma can occur with the conservative approach and no longer observed on MRI or CT $(6,14)$.

\section{Catheter Revision or Replacement}

Many clinicians have chosen to revise or remove the catheter even if the patient is asymptomatic. In the symptomatic patient, the most judicious clinical step should be to immediately stop the intrathecal infusion of the offending agent and choose an alternative method of pain control such as an intravenous infusion until surgical correction can be accomplished. If no major neurological findings are seen on exam such as bladder or bowel function changes or significant motor loss, percutaneous catheter revision or replacement may be performed. Catheter revision can be accomplished by catheter replacement or by simply moving the indwelling catheter caudally one to two interspaces. Catheter replacement or revision should occur with an awake, responsive patient. If the patient experiences paraesthesias as tension is placed on the catheter the procedure should be aborted and surgical decompression should be scheduled. If catheter revision is performed a different drug should be infused post operatively (6).

The physician may choose to totally remove the catheter and pump. If this is the treatment of choice an alternative method of pain relief must be planned. In many patients the pump system was placed as the last option in the treatment algorithm. If this is the case pump removal may not be a reasonable option.

\section{Surgical Decompression and Removal}

If a surgical lesion is suspected, the pump infusion should be stopped immediately and the patient should be referred to a spine surgeon. Resection of the granuloma can be difficult and may require microscopic decompression. Surgical lesions should be suspected if significant motor dysfunction occurs, if the patient experiences loss of bladder function or 
change in bowel function.

\section{Recommendations for Avoiding GRANULOMA}

Based on currently available literature and reported cases, a few recommendations can be made to reduce the risk of granuloma.

\section{Catheter Type and Location}

Multiple exit catheters may have an advantage over single exit catheters. The catheter material has not shown to be a significant cause of granuloma formation, however to date no reported cases have occurred with wire-reinforced catheters. The majority of cases have involved the thoracic spine, however if the physician plans to use lipophilic drugs the catheter tip must be near the pain generator. The level of placement of the catheter tip is a clinical judgment.

\section{Infused Drug}

The Food and Drug Administration has approved only preservative free morphine as the drug of choice for chronic pain of cancer and non-cancer causes (1). Over time many patients develop tolerance and require dose escalation with morphine. In these situations patients may require increasing concentrations of morphine to keep the refill intervals reasonable. In many reported cases of granuloma the concentration has been at or above $40 \mathrm{mgs} / \mathrm{cc}$. If the concentration requirements get to this level alternative strategies should be utilized. In surveys of implanting physicians, up to $35 \%$ of patients using morphine alone fail the therapy. The patients fail because of either lack of efficacy or intolerable side effects. In these clinical situations other drugs such as hydromorphone, fentan$\mathrm{yl}$, clonidine, bupivacaine, and sufentanil may be acceptable alternatives (10). Another strategy is to add a second or third drug to morphine to obtain a synergistic effect and reduce the dosing and concentration requirements of morphine. Similar recommendations can be made when using hydromorphone, since available information also shows a concentration related problem with this drug.

\section{A Surveillance Report of Chronic Pain Patients with Intrathecal Drug DeLIVERY}

\section{Profile of Practice}

The data reported in this study were derived from a large private practice treating both cancer and non-cancer patients. All patients having intrathecal therapies undergo a three to five day inpatient trial with an indwelling catheter. End points include pain relief and a favorable side effect profile. For non-cancer patients, functional improvement is also required. Both intrathecal and epidural trials are used in the screening process. In non-cancer patients a psychological profile with approval from the psychologist is required prior to permanent implant. All patients included in this analysis had implanted programmable pumps (SynchroMed, Medtronic Neurological, Minneapolis, Minnesota), and silastic intrathecal catheters (Medtronic Neurological, Minneapolis, Minnesota). In our practice the catheter tip is placed in the area of the presumed pain generator. The majority of catheters are in the area of $\mathrm{T} 9$ to T11. Catheter tip placement ranges from the C3 level in head and neck syndromes to the sacral nerve roots in rectal and pelvic pain.

\section{Methods}

This study involved a prospective surveillance of 208 consecutive patients presenting for pump refill. Informed consent was obtained from all patients. All patients agreed to participate and were informed of risks and benefits related to the study. Patient anonymity was maintained.

A plain film was used to identify the catheter tip. An MRI with T1 weighted images was performed at the catheter tip in 201 of the patients. MRI scanning was accomplished without difficulty in our patients. Pumps were turned off during scanning, and images were obtained at the level of the catheter tip. The magnetic field effect did not damage or dislodge the pumps. Because the catheters are silicone, there is no concern about possible radiofrequency induced heating of the catheter or adjacent tissue.

In the other seven patients a CT myelogram was performed at the catheter tip. A neuro-radiologist familiar with the diagnosis of inflammatory mass read each study. If the reading was uncertain a second neuro-radiologist reviewed the film to obtain a second opinion. Once the diagnosis of granuloma was found the patients underwent counseling regarding treatment options.
RESULTS

A total of 208 patients underwent imaging studies over a period of 34 weeks. Six patients $(3 \%)$ were found to have a significant lesion. After further evaluation five patients were deemed to be totally asymptomatic with no change in pain levels, physical exam, drug requirements or other clinical factors. One patient was found to have some pain in a dermatomal fashion at the level of the catheter tip. He had not complained of this problem prior to being scheduled for the MRI. The average duration of drug infusion in the group with granulomas was 27 months (range of drug exposure of 3 to 64 months), whereas the average time of exposure in the patients without granulomas was 53 months (range of 2 to 101 months). The average infusion times between groups were not statistically significant, and there was considerable overlap.

All six patients had percutaneous catheter revisions without complication. Each patient chose to have the intrathecal pump continued and was changed from the drug used at the time of diagnosis to a different drug. Since the time of the surgical revision each patient has undergone follow-up images. There have been no recurrent granulomas detected during the 6 to 14 months of follow-up after catheter revisions.

In the group with granulomas, drugs used included morphine, hydromorphone, fentanyl, bupivacaine and clonidine. Three patients received morphine as the only opioid prior to the diagnosis. The maximum concentrations of morphine used in these infusions were $10 \mathrm{mg} / \mathrm{cc}$, $25 \mathrm{mg} / \mathrm{cc}$, and $50 \mathrm{mg} / \mathrm{cc}$. Two patients had hydromorphone as the only opioid prior to the diagnosis. The maximum concentrations used in these patients were $2 \mathrm{mg} /$ $\mathrm{cc}$ and $10 \mathrm{mg} / \mathrm{cc}$. One patient was treated with morphine, hydromorphone, and fentanyl at various times. The maximum concentrations of these drugs were $40 \mathrm{mg} /$ cc, $10 \mathrm{mg} / \mathrm{cc}$ and $2000 \mathrm{mcg} / \mathrm{cc}$, respectively. Three patients had clonidine included in the infusion at the time of diagnosis, however the drug was not used for the entire time of infusion. Similar infusion drugs, doses and protocols were used for patients without granulomas.

\section{ConcLusion}

This intrathecal granuloma surveillance study represents the largest analy- 
sis of patients with intrathecal pumps to date. The finding of a $3 \%$ occurrence of granulomas on diagnostic imaging suggests that the problem may be more prevalent than previously thought. The average duration of the infusions in patients with granulomas was 27 months. The observation that most patients with granulomas were asymptomatic suggests that granulomas develop slowly over time.

MRI scanning was accomplished without difficulty in our patients. Pumps were turned off during scanning and images were obtained at the level of the catheter tip. The magnetic field effect did not damage or dislodge the pumps. The silicone catheters used with the infusion pumps are not subject to radiofrequencyinduced heating during MRI.

Although our literature review suggests that intrathecal catheter granulomas are relatively rare, a finding confirmed by our surveillance study, it remains important to follow patients with intermittent examinations and a thorough review of clinical information. Clearly, most granulomas are asymptomatic.

However, if clinical changes are noted, the physician should have a low threshold for obtaining an imaging study. The decision to remove or revise a catheter after finding a granuloma requires consideration of a number of clinical issues, including whether or not the catheter is symptomatic and the remaining analgesic alternatives available to the patient. Based on the overall low yield of granulomas, we suggest that cost considerations preclude routine imaging in all patients with intrathecal pumps.

Author Affiliation:
Timothy R. Deer, MD
Director and CEO, The Center for
Pain Relief
Charleston, West Virginia and Clini-
cal Faculty
West Virginia University School of
Medicine
Charleston, West Virginia 25301
E-mail: jgoodwin@centerforpainre
lief.com

\section{ReFERENCES}

1. Deer T, Winkelmuller W, Erdine $S$ et al. Intrathecal therapy for cancer and nonmalignant pain: patient selection and patient management. Neuromodulation 1999; 2:55-66.

2. Penn RD. Intrathecal medication delivery. Neurosurg Clin N Am 2003; 14:381-387.

3. North R, Cutchis P, Epstein J et al. Spinal cord compression complicating subarachnoid infusion of morphine: case report and laboratory experience. Neurosurgery 1991; 29:778-784.

4. Coffey R, Burchiel K. Inflammatory mass lesions associated with intrathecal drug infusion catheters: report and observations on 41 patients. Neurosurgery 2002; 50:78-87.

5. McMillan M, Doud T, Nugent W. Catheter-associated masses in patients receiving intrathecal analgesic therapy. Anesth Analg 2003; 96:186-190.

6. Hassenbush S, Burchiel K, Coffey R et al. Management of intrathecal catheter-tip inflammatory masses: a consensus statement. Pain Medicine 2002; 3:313-323.

7. Yaksh T, Hassenbush S, Burchiel K et al. Inflammatory masses associated with intrathecal drug infusion: a review of preclinical evidence and human data. Pain Medicine 2002; 3:300-312.

8. Bidlack J, Hemmick L. Morphine enhancement of mitogen-induced T-cell proliferation. Prog Clin Biol Res 1990; 328:405408.

9. Chuang L, Killam K, Chuang R. Opioid dependency and T-helper cell functions in Rhesus Monkeys. In Vivo 1993; 7:159-166.

10. Bennet G, Serafini M, Burchiel K et al. Evidence based review of the literature on intrathecal delivery of pain medication. J Pain Symptom Manage 2000; 20:S12-S36.

11. Hassenbush S, Stanton-Hicks M, Coving ton $\mathrm{E}$ et al. Long term intraspinal infusions of opioids in the treatment of neuropathic pain. J Pain Symptom Manage 1995; 10:
527-543.

12. Thomsen C, Stahlberg F, Stubgard M et al. Fourier analysis of cerebrospinal fluid flow velocities: MR imaging study. The Scandinavian Flow Group. Radiology 1990; 177: 659-665.

13. Schuchard M, Lanning R, North $R$ et al. Neurological sequelae of intraspinal drug delivery systems: Results of a survey of American implanters of implantable drug delivery systems. Neuromodulation 1998; 1:137-148.

14. Anderson S, Orbegozo M, Racz G et al. Intrathecal granuloma in patients receiving high-dose intrathecal morphine therapy: A report of two cases. Pain Practice 2001; 1: 61-67.

15. Du Pen S. Verbal communication at the intrathecal mass consensus conference. San Francisco, California. February 2002.

16. Perrin J. Unsafe activities of compounding pharmacists. Am J Health Syst Pharm 1995; 52:2827-2828.

17. Medtronic Neurological, Minneapolis, Minnesota. Manufacturer's bulletin. Implanting physician advisory: Important message regarding the occurrence of inflammatory masses at the tip of intraspinal catheters. January 19, 2001.

18. An Expert Consensus Panel on Intrathecal Drug Delivery: The Polyanalgesic Initiative Update 2003, Boston, Massachusets. November, 2003

19. Horais K. Hruby V. Rossi S et al. Effects of chronic intrathecal infusion of a partial differential opioids agonist in dogs. Toxical Sci 2003; 71:263-275.

20. Bejjani GK, Karim NO, Tzortzidis F. Intrathecal granuloma after implantation of a morphine pump: Case report and review of the literature. Surg Neurol 1997; 48: 288-291.

21. Yaksh TL, Horais KA, Tozier NA et al. Chronically infused intrathecal morphine in dogs. Anesthesiology 2003; 99:174-187. 